\title{
Using machine learning of clinical data to diagnose COVID-19: a systematic review and meta-analysis
}

Wei Tse $\mathrm{Li}^{1,2+}$, Jiayan Ma ${ }^{1,2+}$, Neil Shende ${ }^{1,2 \dagger}$, Grant Castaneda ${ }^{1,2}$, Jaideep Chakladar ${ }^{1,2}$, Joseph C. Tsai ${ }^{1,2}$, Lauren Apostol ${ }^{1,2}$, Christine O. Honda ${ }^{1,2}$, Jingyue Xu ${ }^{1,2}$, Lindsay M. Wong ${ }^{1,2}$, Tianyi Zhang ${ }^{1,2}$, Abby Lee ${ }^{1,2}$, Aditi Gnanasekar ${ }^{1,2}$, Thomas K. Honda ${ }^{1,2}$, Selena Z. Kuo ${ }^{3}$, Michael Andrew Yu4, Eric Y. Chang ${ }^{5,6}$, Mahadevan "Raj" Rajasekaran ${ }^{7,8}$ and Weg M. Ongkeko ${ }^{1,2^{*}}$ (i)

\begin{abstract}
Background: The recent Coronavirus Disease 2019 (COVID-19) pandemic has placed severe stress on healthcare systems worldwide, which is amplified by the critical shortage of COVID-19 tests.

Methods: In this study, we propose to generate a more accurate diagnosis model of COVID-19 based on patient symptoms and routine test results by applying machine learning to reanalyzing COVID-19 data from 151 published studies. We aim to investigate correlations between clinical variables, cluster COVID-19 patients into subtypes, and generate a computational classification model for discriminating between COVID-19 patients and influenza patients based on clinical variables alone.
\end{abstract}

Results: We discovered several novel associations between clinical variables, including correlations between being male and having higher levels of serum lymphocytes and neutrophils. We found that COVID-19 patients could be clustered into subtypes based on serum levels of immune cells, gender, and reported symptoms. Finally, we trained an XGBoost model to achieve a sensitivity of $92.5 \%$ and a specificity of $97.9 \%$ in discriminating COVID-19 patients from influenza patients.

Conclusions: We demonstrated that computational methods trained on large clinical datasets could yield ever more accurate COVID-19 diagnostic models to mitigate the impact of lack of testing. We also presented previously unknown COVID-19 clinical variable correlations and clinical subgroups.

Keywords: COVID-19, Machine learning, Diagnostic model

\footnotetext{
* Correspondence: rongkeko@health.ucsd.edu

${ }^{+}$Wei Tse Li, Jiayan Ma and Neil Shende contributed equally to this work.

'Department of Surgery, Division of Otolaryngology-Head and Neck Surgery,

UC San Diego School of Medicine, San Diego, CA 92093, USA

${ }^{2}$ Research Service, VA San Diego Healthcare System, San Diego, CA 92161,

USA

Full list of author information is available at the end of the article
}

(c) The Author(s). 2020 Open Access This article is licensed under a Creative Commons Attribution 4.0 International License, which permits use, sharing, adaptation, distribution and reproduction in any medium or format, as long as you give appropriate credit to the original author(s) and the source, provide a link to the Creative Commons licence, and indicate if changes were made. The images or other third party material in this article are included in the article's Creative Commons licence, unless indicated otherwise in a credit line to the material. If material is not included in the article's Creative Commons licence and your intended use is not permitted by statutory regulation or exceeds the permitted use, you will need to obtain permission directly from the copyright holder. To view a copy of this licence, visit http://creativecommons.org/licenses/by/4.0/. The Creative Commons Public Domain Dedication waiver (http://creativecommons.org/publicdomain/zero/1.0/) applies to the data made available in this article, unless otherwise stated in a credit line to the data. 


\section{Background}

COVID-19 is a severe respiratory illness caused by the virus SARS-CoV-2. The scientific community has focused on this disease with near unprecedented intensity. However, the majority of primary studies published on COVID-19 suffered from small sample sizes [1, 2]. While a few primary research studies reported on dozens or hundreds of cases, many more studies reported on less than 20 patients $[3,4]$. Therefore, there is an urgent need to collate all available published data on the clinical characteristics of COVID-19 from different studies to construct a comprehensive dataset for gaining insights into the pathogenesis and clinical characteristics of COVID-19. In this study, we aim to perform a large-scale meta-analysis to synthesize all published studies with COVID-19 patient clinical data, with the goal of uncovering novel correlations between clinical variables in COVID-19 patients. We will then apply machine learning to reanalyze the data and construct a computational model for predicting whether someone has COVID-19 based on their clinical information alone.

We believe that the ability of predicting COVID-19 patients based on clinical variables and using an easily accessible computational model would be extremely useful to address the widespread lack of testing capabilities for COVID-19 worldwide. Because many countries and hospitals are not able to allocate sufficient testing resources, healthcare systems are deprived of one of their most effective tools for containing a pandemic: identification of case hotspots and targeted action towards regions and specific individuals with the disease [5]. The scale of the testing shortage calls for methods for diagnosing COVID-19 that use resources local healthcare facilities currently have. We propose the development of a disease prediction model based on clinical variables and standard clinical laboratory tests.

A number of meta-analyses have been done on COVID19 , but almost none of them comprehensively included data from all published studies. Three different meta-analyses, published in February, March, and April of 2020, included data from 10, 8, and 31 articles, respectively [6-8]. We included 151 articles, comprising 413 patients, in our analysis. To the best of our knowledge, no study has performed a large-scale machine learning analysis on clinical variables to obtain a diagnostic model. We believe that our study will be an important step towards leveraging the full extent of published clinical information on COVID-19 patients to inform diagnosis of COVID-19, instead of relying on general guidelines for symptoms that do not take into account the association between different clinical variables.

\section{Materials and methods}

Literature search and inclusion criteria for studies

Patient clinical data were manually curated from a PubMed search with the keyword "COVID-19." A total of 1439 publications, dating from January 17, 2020 to March 23, 2020, were reviewed. All publications with no primary clinical data, including reviews, meta-analyses, and editorials, were excluded from our analysis. After manual review, we found 151 studies with individual-level data, encompassing data from 413 patients. All individual patient data with 2 more clinical variables reported per patient were included. Clinical variables sought for included demographics, signs and symptoms, laboratory test results, imaging results, and COVID-19 diagnosis. The compiled dataset with clinical variables for each patient, along with a reference to the source study for each patient, can be found in Table S4 and in the following repository: https:/github.com/yoshihiko1218/COVID19ML/projects.

For our machine learning classification task to discriminate COVID-19 patients from influenza patients, we used clinical variables for 21 influenza patients from a study by Cheng et al. and 1050 patients from the Influenza Research Database $[9,10]$. Only H1N1 Influenza A virus cases were included because of difficulties locating data from other strains.

\section{Correlational tests between pairs of clinical variables}

We sought to uncover correlations that could yield critical insights into the clinical characteristics of COVID19 by correlating every variable to each other. For two continuous variables, the Spearman correlation test was applied. For one continuous variable and one categorical variable, the Kruskal-Wallis test was applied. For two different categorical variables, the chi-squared test was applied. All statistical tests were considered significant if the $p$-value is 0.05 or below.

\section{Machine learning for classification of COVID-19 patients into subtypes}

A self-organizing map (SOM) is an artificial neural network that constructs a two-dimensional, discretized depiction (map) of the training set. We used the SOM algorithm to cluster our patients based on similar patterns of clinical variables. The SOMbrero package in $\mathrm{R}$ was used [11]. Because clustering of neurons are performed using Euclidean Distance, we first standardized each clinical variable to ensure that they are equally weighted.

The trainSOM function was used to implement numeric SOM on our data set, which is inputted as an $\mathrm{N} \mathrm{x}$ P matrix, with $N=398$ patients and $P=48$ variables. From this, we selected 27 clinical variables with very high significativity $(p<0.001)$ after running an ANOVA test across all neurons and ran another iteration of trainSOM with these variables. We generated SOMs from the $3 \times 3$ neuron grid to $20 \times 20$ neuron grid and selected the $9 \times 9$ SOM with 81 neurons as our final model based on minimal topographic error. We then aggregated the 
neurons into super-clusters using the superClass method in SOMbrero.

\section{Preprocessing of data for machine learning classification} Data were preprocessed by combining data from COVID19 cases and influenza cases into a single matrix, followed by removal of any clinical variables that were not present in both the COVID-19 dataset and the influenza dataset. Nineteen clinical variables were included as machine learning input. The variables include age, sex, serum levels of neutrophil (continuous and ordinal), serum levels of leukocytes (continuous and ordinal), serum levels of lymphocytes (continuous and ordinal), result of CT scans, result of chest $\mathrm{X}$-rays, reported symptoms (diarrhea, fever, coughing, sore throat, nausea, and fatigue), body temperature, and underlying risk factors (renal diseases and diabetes). Categorical data were converted to dummy variables using the get dummies function in Pandas because non-numerical data are not allowed in our machine learning algorithm.

\section{Performing XGBoost classification}

The eXtreme Gradient Boosting algorithm (XGBoost), an ensemble machine learning method widely known for its superior performance over other machine learning methods, was selected for our study [12]. We first split our data into $80 \%$ training dataset and $20 \%$ testing dataset. 5 -fold cross-validation was then performed, with 70 boosting rounds (iterations), and fed into a Bayesian optimization function for calculation of the best hyperparameters for XGBoost. The hyperparameters tuned included max depth, gamma, learning rate, and n_estimators. Bayesian optimization was performed with an initial 8 steps of random exploration followed by 5 iterations. The expected improvement acquisition function was used. We also performed XGBoost classification on subgroups of COVID-19 patients, stratifying them by gender, age, and SOM superclusters. For each gender and age subgroup, only influenza patients of the corresponding age and gender are included.

\section{Evaluation of classification results}

XGBoost results were evaluated by plotting a receiver operating characteristic (ROC) curve and a precision recall (PR) curve. The area under the curve (AUC) was also calculated for both curves. We also performed classification using three other machine learning models, LASSO, RIDGE, and random forest, and compared results obtained with that obtained by XGBoost. AUC of the ROC curve was compared across the different models.

\section{Results}

\section{Compilation of patient data and summary of clinical variables}

After compiling information from 151 published studies, we present a total of 42 different clinical variables, including 21 categorical and 21 continuous variables, that are reported in more than 1 study. Discrete variables include nominal categorical variables like gender, which is 49.49\% (194 patients) male and 50.51\% (198 patients) female, and ordinal categorical variables like lymphocytes level, of which 86 patients (48.86\%) have low levels, 73 patients $(41.47 \%)$ have normal levels, and 17 patients (9.65\%) have high levels. Continuous variables include age, which has a mean of 38.91 years and variance 21.86 years, and serum neutrophil levels, which has a mean of $6.85 \times 10^{9}$ cells $/ \mathrm{L}$ and a variance of $12.63 \times 10^{9}$ cells $/ \mathrm{L}$. Certain variables, including all counts of blood cell populations, have both ordinal and continuous components. The continuous component describes the raw count of these populations, while the ordinal component describes whether these counts are within normal range, below normal range, or above normal range. A summary for all data is shown in Table 1.

To evaluate heterogeneity of patient data between the different studies, we performed principle component analysis (PCA) using all clinical variables as input (Figure S1). Visualization of both $\mathrm{PC} 1$ vs. $\mathrm{PC} 2$ and $\mathrm{PC} 2$ vs. PC3 revealed no significant heterogeneity between the different studies.

\section{Relationship between pairs of clinical variables}

We performed correlation between all possible pairs of clinical variables to uncover potentially important associations (Table S1). If both variables are continuous, the Spearman correlation test is applied $(p<0.05)$. Among 143 Spearman correlation tests, 27 show significant correlation, with 9 of these involving age, corroborating reports that age plays a critical role in the development of COVID-19 [13]. We observed $C$ reactive protein (CRP) levels and serum platelets levels to be the variables with the strongest correlations with age (Fig. 1a). CRP levels, an indicator of inflammation, are positively correlated with increasing age, while platelets levels are negatively correlated with increasing age. Other than age, we observed a negative correlation between the levels of CRP and lymphocytes levels and a positive correlation between CRP levels and neutrophil levels (Fig. 1a). This result suggests that inflammation is most likely driven by neutrophils. The serum levels of white blood cells are also strongly correlated with neutrophils, further suggesting that white blood cell counts are heavily influenced by neutrophil levels (Fig. 1a).

For pairs of one continuous and one discrete variable, the Kruskal-Wallis test is applied $(p<0.05)$. Among 319 Kruskal-Wallis comparisons, 36 correlations were significant. Some of the significant pairs overlapped with correlations between two continuous variables for variables that have both ordinal and continuous components. Such correlations are not displayed twice in Fig. 1. We found again that age correlated significantly with multiple variables, including negative correlation with lymphocyte 
Table 1 Clinical Variables Summary of Meta-analysis

\begin{tabular}{|c|c|c|c|c|}
\hline \multicolumn{5}{|l|}{ Continuous Variable } \\
\hline Clinical Variable & \# of Data & mean & median & variance \\
\hline Age & 389 & 38.91306 & 39 & 21.85783 \\
\hline NumberOfFamilyMembersInfected & 54 & 3.37037 & 2 & 2.6338 \\
\hline neutrophil & 103 & 6.854078 & 3.31 & 12.62838 \\
\hline SerumLevelsOfWhiteBloodCell & 130 & 7.031223 & 5.965 & 4.250785 \\
\hline lymphocytes & 135 & 2.022841 & 0.98 & 4.207139 \\
\hline Plateletes & 50 & 220.32 & 185.5 & 146.3334 \\
\hline CReactiveProteinLevels & 139 & 31.18187 & 15 & 40.4953 \\
\hline Eosinophils & 8 & 0.06125 & 0.01 & 0.070078 \\
\hline RedBloodCells & 4 & 4.225 & 4.205 & 0.189011 \\
\hline Hemoglobin & 24 & 45.5 & 14.5 & 49.99953 \\
\hline Procalcitonin & 33 & 2.586394 & 0.07 & 12.54482 \\
\hline DurationOflllness & 88 & 14.06818 & 12 & 8.970653 \\
\hline DaysToDeath & 3 & 12.66667 & 12 & 6.548961 \\
\hline DaysBeforeSymptomsAppear & 38 & 7.368421 & 6 & 5.142297 \\
\hline NumberOfAffectedLobes & 24 & 1.75 & 2 & 1.163687 \\
\hline TimeBetweenAdmissionAndDiagnosis & 47 & 5.893617 & 6 & 4.116568 \\
\hline bodyTemperature & 67 & 37.6209 & 37.5 & 0.972999 \\
\hline Hematocrit & 7 & 0.320286 & 0.355 & 0.078175 \\
\hline ActivatedPartialThromboplastinTime & 9 & 33.18889 & 33.4 & 3.642784 \\
\hline fibrinogen & 9 & 3.685556 & 3.91 & 0.752184 \\
\hline urea & 19 & 3.123158 & 3 & 0.863884 \\
\hline \multicolumn{5}{|l|}{ Discrete Variable } \\
\hline Variables & & Number & & Percentage \\
\hline \multicolumn{5}{|l|}{ Sex } \\
\hline M & & 194 & & 49.4898 \\
\hline $\mathrm{F}$ & & 198 & & 50.5102 \\
\hline \multicolumn{5}{|l|}{ Community Transmission } \\
\hline Yes & & 93 & & 37.5 \\
\hline No & & 46 & & 18.54839 \\
\hline No/Wuhan & & 109 & & 43.95161 \\
\hline \multicolumn{5}{|l|}{ Neutrophil } \\
\hline low & & 15 & & 11.81102 \\
\hline normal & & 83 & & 65.35433 \\
\hline high & & 29 & & 22.83465 \\
\hline \multicolumn{5}{|l|}{ Serum Levels Of White Blood Cell } \\
\hline low & & 55 & & 32.35294 \\
\hline normal & & 94 & & 55.29412 \\
\hline high & & 21 & & 12.35294 \\
\hline \multicolumn{5}{|l|}{ Lymphocytes } \\
\hline low & & 86 & & 48.86364 \\
\hline normal & & 73 & & 41.47727 \\
\hline high & & 17 & & 9.659091 \\
\hline
\end{tabular}


Table 1 Clinical Variables Summary of Meta-analysis (Continued)

\begin{tabular}{|c|c|c|c|c|}
\hline \multicolumn{5}{|c|}{ Continuous Variable } \\
\hline Clinical Variable & \# of Data & mean & median & variance \\
\hline normal & & 60 & & 37.97468 \\
\hline high & & 98 & & 62.02532 \\
\hline \multicolumn{5}{|l|}{ CT Scan Results } \\
\hline pos & & 124 & & 89.20863 \\
\hline neg & & 15 & & 10.79137 \\
\hline \multicolumn{5}{|l|}{ RT-PCR Results } \\
\hline pos & & 100 & & 96.15385 \\
\hline neg & & 4 & & 3.846154 \\
\hline \multicolumn{5}{|l|}{ X-ray Result } \\
\hline pos & & 35 & & 74.46809 \\
\hline neg & & 12 & & 25.53191 \\
\hline \multicolumn{5}{|l|}{ GGO } \\
\hline Yes & & 92 & & 96.84211 \\
\hline No & & 3 & & 3.157895 \\
\hline \multicolumn{5}{|l|}{ Diarrhea } \\
\hline Yes & & 30 & & 45.45455 \\
\hline No & & 36 & & 54.54545 \\
\hline \multicolumn{5}{|l|}{ Fever } \\
\hline Yes & & 261 & & 91.25874 \\
\hline No & & 25 & & 8.741259 \\
\hline \multicolumn{5}{|l|}{ Coughing } \\
\hline Yes & & 164 & & 82.82828 \\
\hline No & & 34 & & 17.17172 \\
\hline \multicolumn{5}{|l|}{ Shortness Of Breath } \\
\hline Yes & & 45 & & 60 \\
\hline No & & 30 & & 40 \\
\hline \multicolumn{5}{|l|}{ Sore Throat } \\
\hline Yes & & 37 & & 60.65574 \\
\hline No & & 24 & & 39.34426 \\
\hline \multicolumn{5}{|l|}{ Nausea/Nomiting } \\
\hline Yes & & 18 & & 52.94118 \\
\hline No & & 16 & & 47.05882 \\
\hline \multicolumn{5}{|l|}{ Pregnant } \\
\hline Yes & & 43 & & 66.15385 \\
\hline No & & 22 & & 33.84615 \\
\hline \multicolumn{5}{|l|}{ Fatigue } \\
\hline Yes & & 8 & & 61.53846 \\
\hline No & & 5 & & 38.46154 \\
\hline
\end{tabular}

levels, positive correlation with neutrophil levels, and positive correlation with shortness of breath (Fig. 1b). Other interesting associations were also discovered. Coughing was found to be correlated with increasing fibrinogen levels and decreasing lymphocyte levels. Those with lower levels of serum white blood cells (leukocytes) are more likely to report a positive CT scan result for pneumonia. Females may experience a greater number of days before symptoms appear. Finally, we found that sore throat decreases with increasing CRP levels (Fig. 1b). 


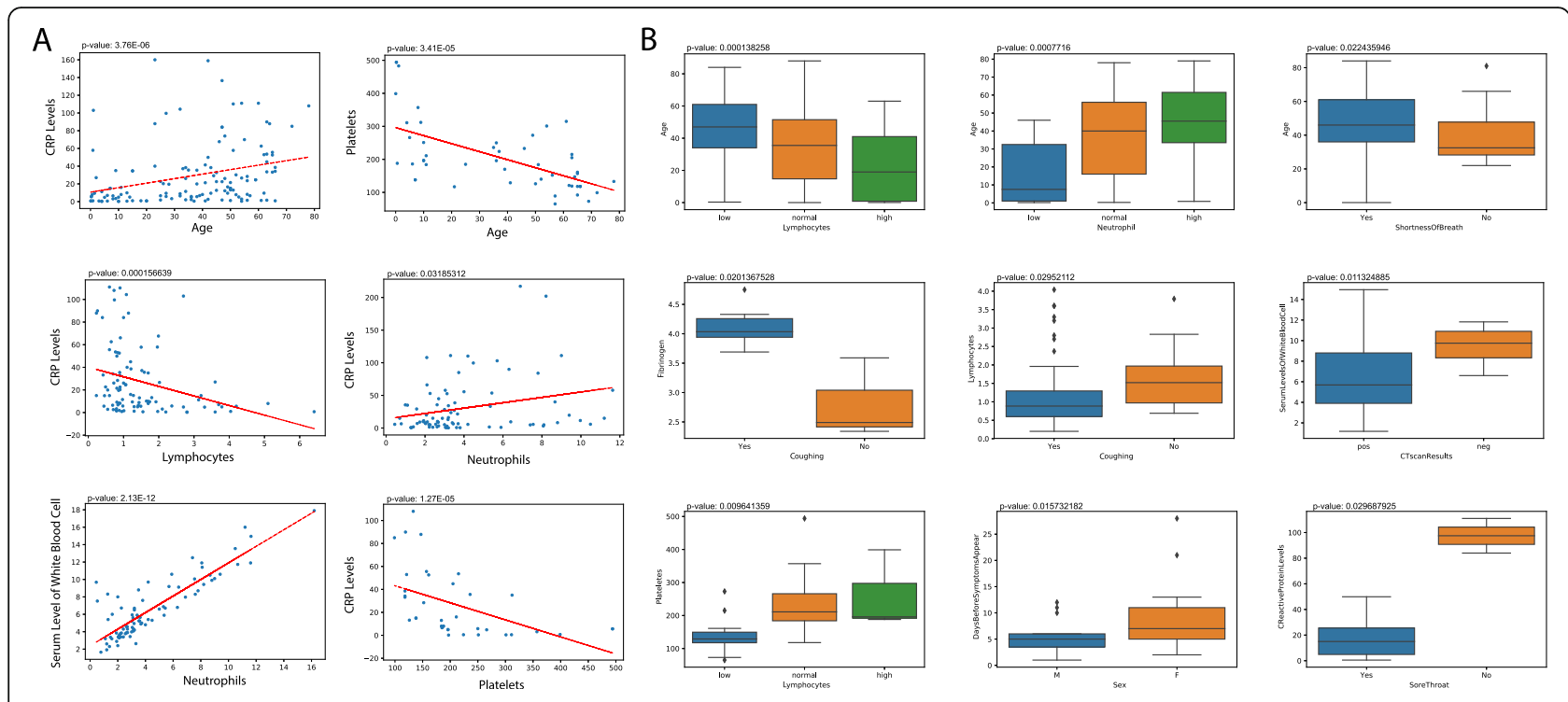

Fig. 1 Select correlations with continuous clinical variables for COVID-19 patients. a Correlations between two continuous variables (Spearman, $p<0.05$ ). b Correlations between one continuous and one categorical variable (Kruskal-Wallis test, $p<0.05$ )

For pairs of two categorical variables, a two-tailed chisquare test is applied. 42 out of 309 comparisons showed significant correlation, with few overlaps with former tests. Gender is involved in 6 of the significant correlation, indicating significant gender differences in COVID-19. Contingency tables of selected significant correlations are shown in Fig. 2. Males were found to have higher lymphocyte and neutrophil levels than females (Fig. 2a,b). Females were found to be more likely to have lower levels of serum white blood cells (Fig. 2c).

\section{Clustering of patients into subcategories of COVID-19}

We next aim to cluster COVID-19 patients based on clinical variables using machine learning. We chose the

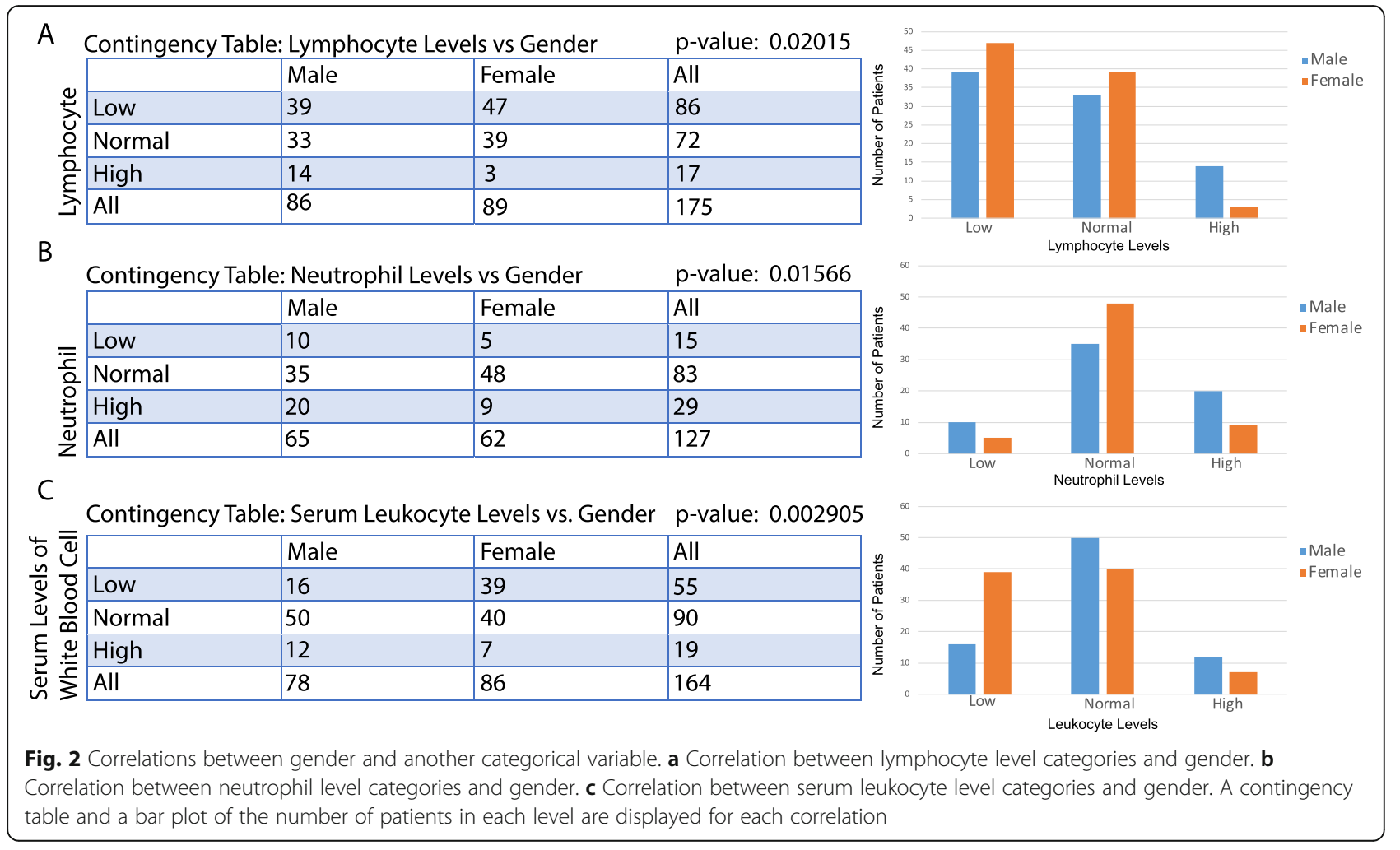


well-known SOM algorithm for clustering. $\mathrm{SOM}$ is a neural network that has a set of neurons organized on a 2D grid [14]. All neurons are connected to all input units (individual patients) by a weight vector. The weights are determined through iterative evaluations of a Gaussian neighborhood function, with the result of creating a 2D topology of neurons to model the similarity of input units (individual patients). The algorithm outputs a map that assigns each sample to one of the neurons on the $2 \mathrm{D}$ grid, with samples in the same neuron being the most similar to one another. Similarity of samples decreases with distance between neurons on the 2D map. Missing variables were ignored from the SOM model when deriving a neural topology.

We generated square SOM neuron grids with side lengths 3 through 20 using the trainSOM function in the $\mathrm{R}$ package SOMbrero. The grids with side lengths 3 , $4,5,7$, and 9 all had topographic errors of 0 (Fig. 3a). Of these, we chose the biggest grid $(9 \times 9=81$ clusters $)$ as our model. After the patients were assigned to neurons, an analysis of variance (ANOVA) test was performed to test which variables actively participate in the clustering. Of the 48 clinical variables we inputted, 27 were found to have very high significativity $(p<0.001)$ (Table S2). We then reran the SOM using the 27 variables on a $9 \times$ 9 grid. This grid is displayed on Fig. $3 \mathrm{~b}$ and has a final energy of 8.139248. The largest neuron has 39 patients, the second largest has 37 , the third largest has 21 , and the fourth largest has 20 (Fig. 3c).

\section{Clinical characteristics of COVID-19 clusters}

We then examined the defining features of patients assigned to the same neurons. We investigated four neurons associated with the largest number of patients and identified the four variables with the smallest nonzero standard deviations for each patient cluster. In the largest cluster, with 39 patients, the four smallest nonzero standard deviations were for the variables region of infection, sore throat, RT-PCR results, and coughing. In the second largest cluster, with 37 patients, the variables were baby death if pregnant, lymphocyte levels, fever, and coughing. In the next largest cluster, with 21 patients, the variables were sore throat, duration of illness in days, RT-PCR results, and coughing. In the fourth largest cluster, with 20 patients, the variables were sore throat, fever, coughing, and age.

We next used the function superClass to compute the relative Euclidean distances between the 81 patient clusters and form superclusters. The relative distances between the individual clusters are shown in Fig. 3d-e. We divided the 81 clusters into 4 superclusters, which are represented in Fig. 3b by the color of the squares. Supercluster 1 was formed with 24 neurons, supercluster 2 had 28 neurons, supercluster 3 had 12, and supercluster
4 had 17 neurons. Visualizing distances between neighboring neurons, we found that the distances are the smallest at corners of the grid, especially the upper right-hand corner (Fig. 3f). This corner corresponds to supercluster 4, suggesting that patients within this cluster may be especially similar.

Next, we sought to determine the clinical features that effectively distinguish these superclusters. We performed Kruskal-Wallis testing on the values of the 27 variables across the four superclusters. Twenty-four variables were significantly different between the superclusters $(p<$ $0.05)$ (Table S3). We discovered that the clinical variables exhibit 3 main types of correlations with the superclusters: continuous increase in value from cluster 1 to cluster 4 (Fig. $3 \mathrm{~g}$ ), clusters 1 and 3 exhibiting the same distribution and clusters 2 and 4 exhibiting another distribution (Fig. 3h), and 3 clusters exhibiting the same median (Fig. 3i). From these analyses, we could infer that patients with low levels of CRP and serum immune cells likely define cluster 1 . Cluster 1 patients are also predominantly female. Cluster 2 contains patients with slightly higher levels of CRP and serum immune cells than cluster 1 . Compared to cluster 1 patients, fewer cluster 2 patient reported coughing and fever. Cluster 2 patients are predominantly male. Cluster 3 contains patients with few reported symptoms, including less coughing, shortness of breath, fever, and sore throat. Cluster 3 is overwhelmingly female. Cluster 4 most likely contains patients not belonging to the other 3 clusters as it has few distinguishing features and high levels of missing data.

\section{Creation of a diagnostic model for COVID-19 based on clinical variables}

Because it can be difficult to distinguish influenza from COVID-19, we downloaded clinical data collected for influenza from a study by Cheng et al. and from the Influenza Research Database [9, 10]. Machine learning was then used to perform a classification task to discriminate between influenza and COVID-19. For machine learning, we employed the algorithm Extreme Gradient Boosting (XGBoost) using Python. XGBoost is a novel, state-of-theart machine learning algorithm that has been shown to outperform other more traditional algorithms in its accuracy and efficiency [12]. It can also take both continuous and discrete inputs and handle sparse data, in addition to having highly optimizable hyper-parameters [15].

The datasets from non-COVID patients and COVID19 patients were merged and then split into training and testing patient sets, with $80 \%$ and $20 \%$ of the patients, respectively. Categorical variables were encoded as dummy variables. We then tuned the model using the Bayesian optimization method for hyperparameter search. We found the best hyperparameters to be 


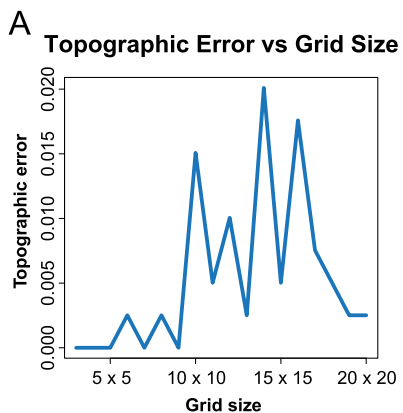

D

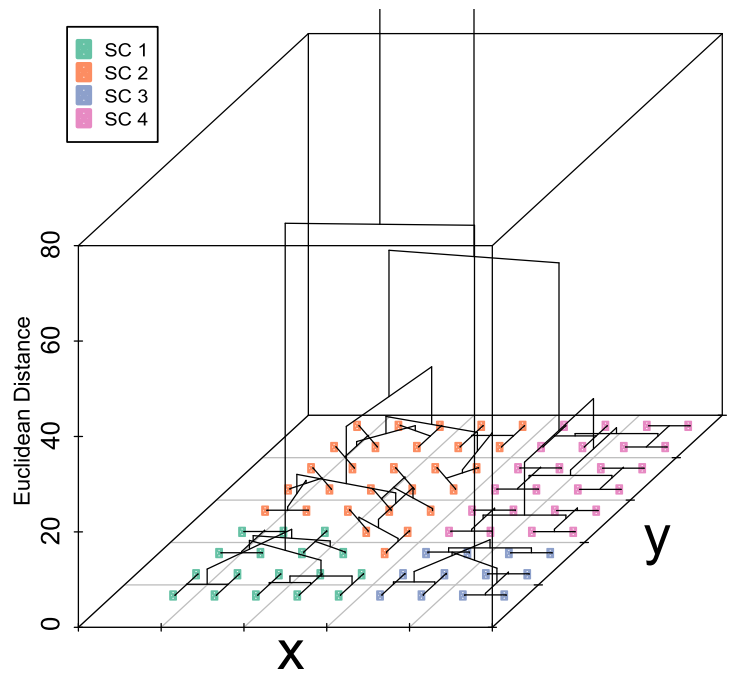

B

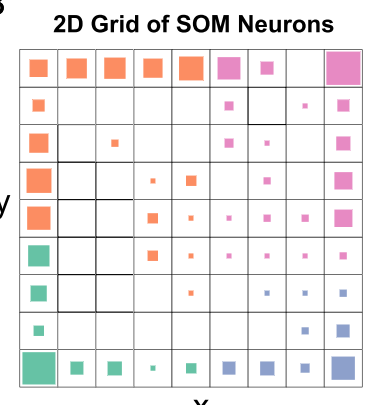

$\mathrm{E}$
C

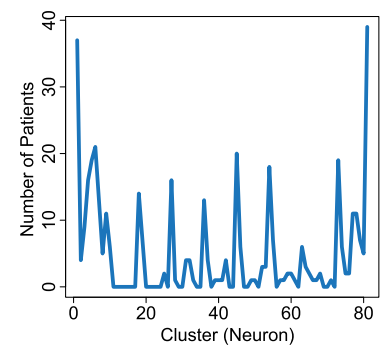

Superclusters dendrogram

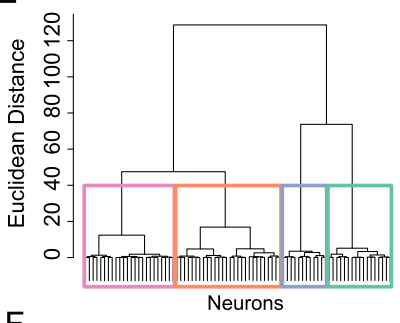

F Gradient Map of Distances

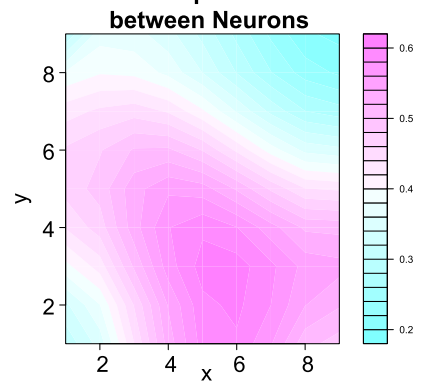

G
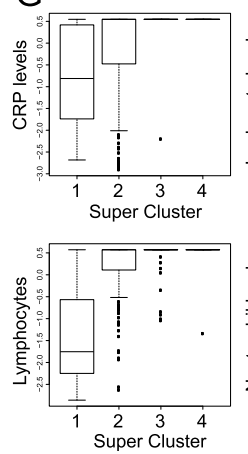

$\mathrm{H}$
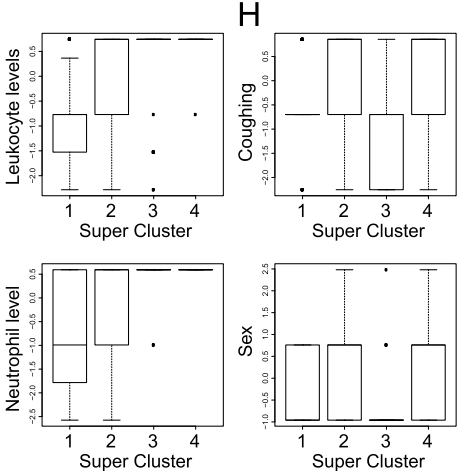
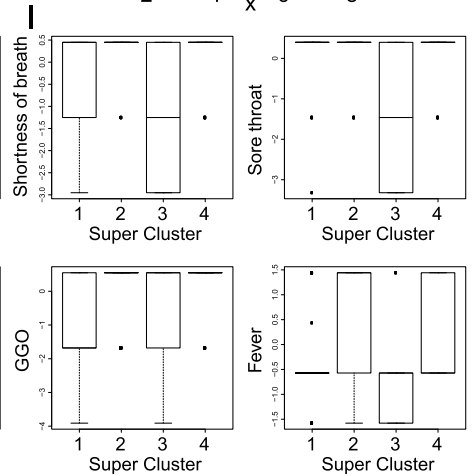

Fig. 3 Summary of COVID-19 patient clustering using SOM. a Plot of topographic error of the 2D SOM grid vs. size of the grid. b 2D plot of SOM neurons after retaining only the most significant clinical variable for analysis. Each small grid represents a neuron, and the size of the square in each grid represents the number of patients associated with each neuron. The color code corresponds to superclusters presented in panel (d). $\mathbf{c}$ Plot of number of patients in each neuron. $\mathbf{d}$ 3D dendrogram summarizing the neurons into superclusters. e 2D dendrogram with the same information as the dendrogram in panel (d). In both dendrograms, the vertical axis represents the relative distance between clusters, which can be known between any two clusters by looking at the branch point where they diverge. $\mathbf{f}$ Gradient map where light blue regions of the SOM depict higher similarity of neurons with each other. $\mathbf{g}$ Boxplots of immune-associated clinical variables that differentiate superclusters. $\mathbf{h}$ Boxplots in which superclusters 1 and 3 display similar trends. i Boxplots in which only one supercluster has a median at a different value from the other three. All variables have been previously normalized. For binary variables, only three possible positions on the vertical axis is possible: the bottom one being no, the middle one being yes, and the top one being missing. For the gender (sex) variable, the bottom position is female, the middle is male, and the top one is missing 
gamma $=0.0933$, learning rate $=0.4068$, $\max$ depth $=$ 6.558 , and n_estimators $=107.242$.

\section{Evaluation of XGBoost classification outcomes}

From the ROC curve of prediction results, we obtained an AUC of 0.990 (Fig. 4a). However, because there is an imbalance of class in our input (i.e. we have significantly more influenza patients than COVID-19 patients), the precision recall $(\mathrm{PR})$ curve may be better able to present our model's results. ROC curves could be significantly influenced by skewing the distribution of classes in classification, while PR curves would not be impacted by this action. We observed a slightly lower AUC of 0.977 in our PR curve and computed the F1 score to be 0.929 (Fig. 4b), which suggest that our model is still highly accurate even when class imbalances are taken into account. The prediction result from XGBoost's predict function was used to plot a confusion matrix (Fig. 4c). From the confusion matrix, we calculated a sensitivity of $92.5 \%$ and a specificity of $97.9 \%$. We found the most important features in our prediction model to be age, CT scan result, temperature, lymphocyte levels, fever, and coughing, in order of decreasing importance (Fig. 4d). We also provided a 6-level decision tree sample of our XGBoost model (Fig. 4e), which is not a representation of our full model.

We applied the XGBoost model to our 4 SOM superclusters to investigate whether classification results are better for specific subtypes of patients. The ROC AUC is over 0.9 for all 4 superclusters, with the best classification performance seen for clusters 3 and 4 (Figure S2). The high AUC for cluster 4 may not be accurate, however, because it contains more missing data fields.
Classification of COVID-19 vs. influenza patients using other machine learning models

Since XGBoost could be prone to overfitting, which is an inherent disadvantage of boosting models, we have also fitted other machine learning classification models to our data. Using RIDGE regression, random forest, and LASSO regression, we have obtained an AUC of 96.6\%, 95.3\%, and $96.3 \%$, respectively, when trying to distinguish between influenza and COVID-19 patients (Fig. 5). With RIDGE regression, the sensitivity was around $87 \%$, and the specificity was around $92 \%$. With random forest, the sensitivity was $100 \%$, and the specificity was around $90 \%$. However, based on the ROC curve, the random forest method does not have great power. With LASSO, the sensitivity was around $85 \%$, while the specificity was around $93 \%$. While none of these models achieved as high of an accuracy as XGBoost, the reasonably high accuracy achieved by these models suggested that XGBoost's classification power is likely not exclusively due to overfitting.

\section{Classification performance based on gender and age groups}

We applied our classification models to five different demographic groups: male, female, young (18-39 years old), middle age (40-65 years old), and old ( $>65$ years old). We discovered that XGBoost performs the best in all classification tasks out of all our models, except for when the model was used for old patients (Fig. 6). For patients 65 years old or above, the classification power was poor for all models. This is likely due to the relatively fewer number of patients in our dataset who are older than 65. Other than this cohort, we found a very high ROC AUC for all other cohorts (Fig. 6).

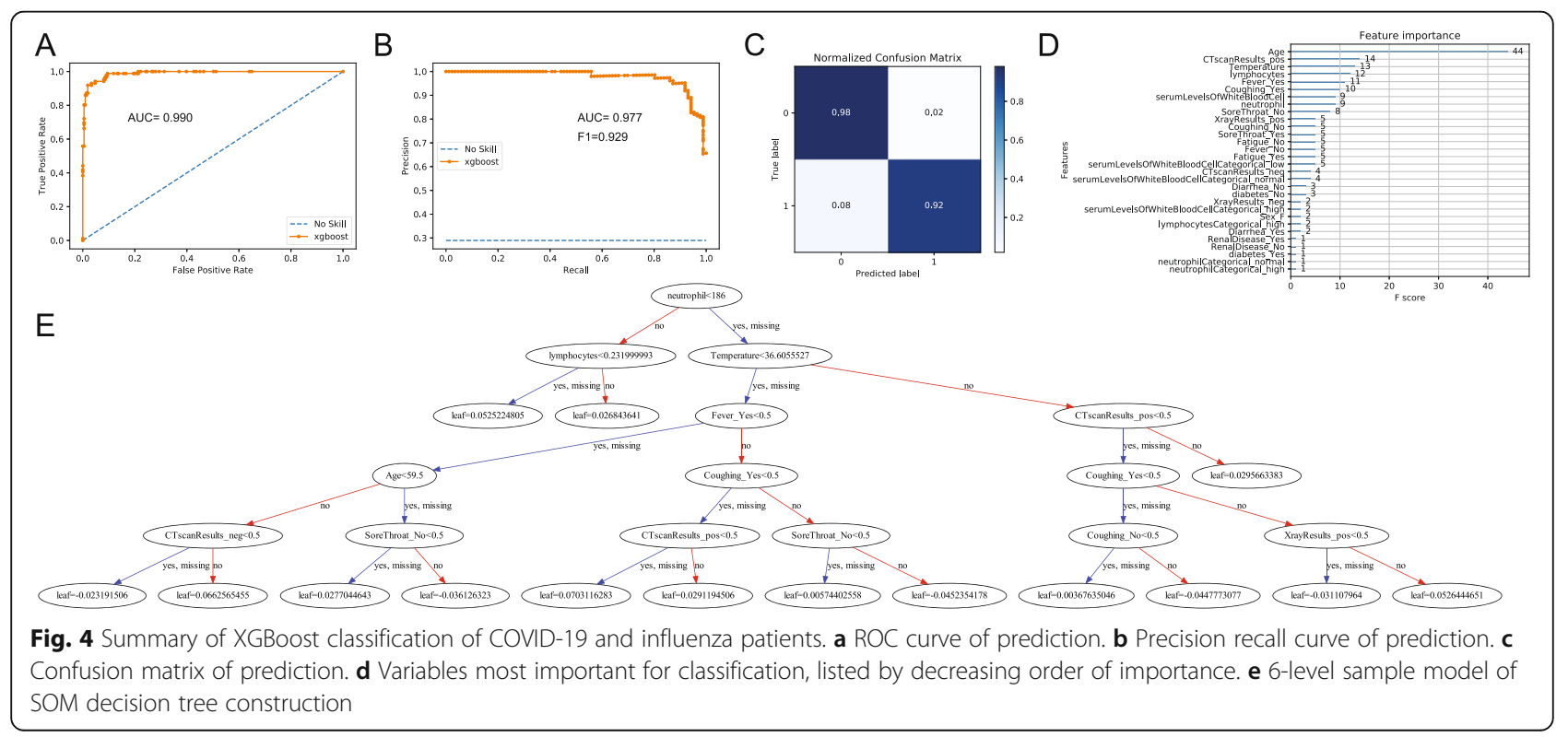



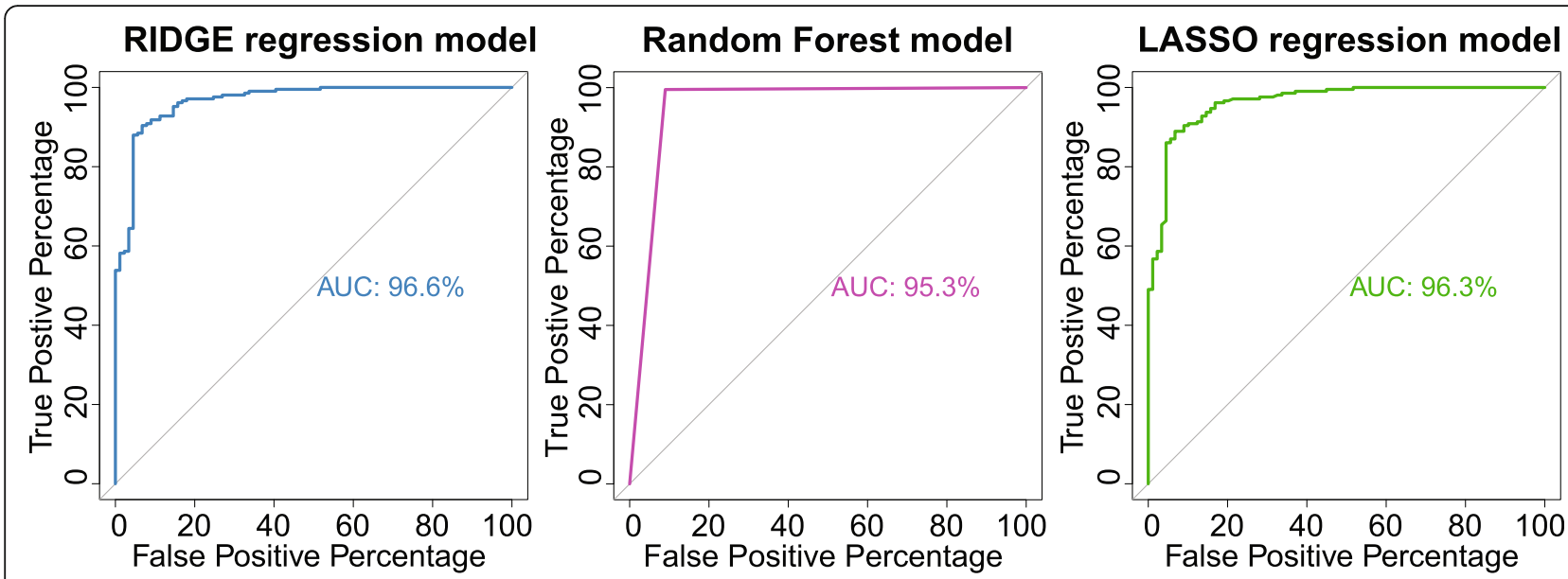

Fig. 5 Classification of COVID-19 vs. influenza patients using RIDGE, random forest, and LASSO models. ROC curves and AUC for each model were presented

\section{Discussion}

As the recent pandemic of COVID-19 unfolds across the world, the inability of countries to test their citizens is heavily impacting their healthcare system's ability to fight the epidemic. Testing is necessary for the identification and quarantine of COVID-19 patients. However, the multi-step process required for the conventional SARS-CoV-2 test, via quantitative polymerase chain reaction (qPCR), is creating difficulties for countries to test large numbers of suspected patients [16]. Testing begins with a healthcare worker taking a swab from the patient. The swab is sent to a laboratory, and viral RNA is extracted from the sample and reverse transcribed into DNA. The DNA is tagged with a fluorescent dye and then amplified using a qPCR machine. If a high level of fluorescence is observed compared to control, the sample is positive with SARS-CoV-2. Each step of the testing process is susceptible to severe shortages [17].

In this study, we aim to mine published clinical data of COVID-19 patients to generate a new diagnostic framework. We hypothesize that novel or complex associations between clinical variables could be exploited for diagnosis with the aid of machine learning. Not only may underlying relationships between clinical variables in COVID-19 be useful for the development of a computational diagnostic test based on signs, symptoms, and laboratory results, these correlations can also yield critical insights into the biological mechanisms of COVID-19 transmission and infection.

Using correlational tests, we corroborated previous findings and expected results for COVID-19 patients but also uncovered novel relationships between clinical variables. We found that age is correlated with CRP level, an indicator of inflammation, and decreased platelet levels. It is known that as age increases, the proinflammatory response becomes stronger, leading to increasing CRP and decreasing platelet levels [18]. However, we found surprising correlations with gender, including higher serum neutrophil and leukocyte levels in males compared to females. According to the National Health and Nutrition Examination Survey, with data from over 5600 individual, few differences exist between male and females in the serum levels of these cells [19]. Another study with 200 samples found that neutrophils are generally higher in women [20]. Correlations with gender observed here may offer a piece of the explanation for why men infected with COVID-19 seem to experience poorer prognosis, one of the important outstanding questions of COVID-19 [21].

We also classified COVID-19 patients into different clusters using the SOM machine learning algorithm. Two of the clusters are defined by low vs. high levels of immunological parameters, including immune cell counts and CRP levels. A third cluster is defined by a tendency for fewer reported symptoms, including sore throat, fever, and shortness of breath, and is predominantly female.

Finally, using the machine learning algorithm XGBoost, we constructed a computational model that successfully classified influenza patients from COVID-19 patients with high sensitivity and specificity. We believe that our model demonstrated the feasibility of using data mining and machine learning to inform diagnostic decisions for COVID-19. Such a model could be extremely useful for more effective identification of COVID-19 cases and hotspots, which could allow health officials to act before testing shortages could be addressed.

Interestingly, we have found age to be the most significant determinant of the accuracy of our model. We hypothesize that this could be due to the respective incidence rates of COVID-19 and influenza for each age group. While COVID-19 disproportionally affects the elderly population, influenza affects the younger 

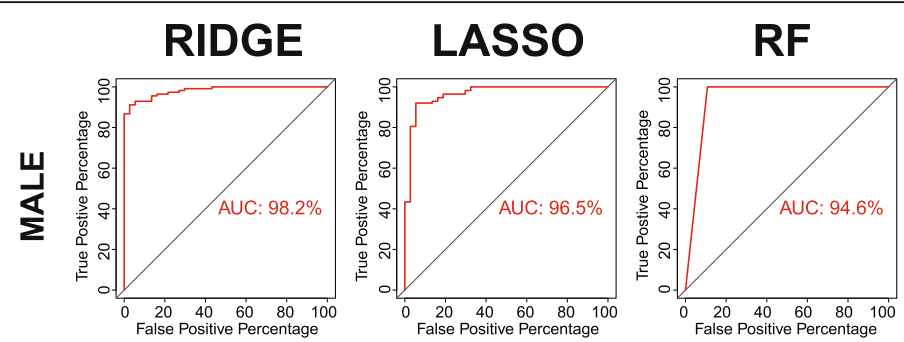

XGBoost
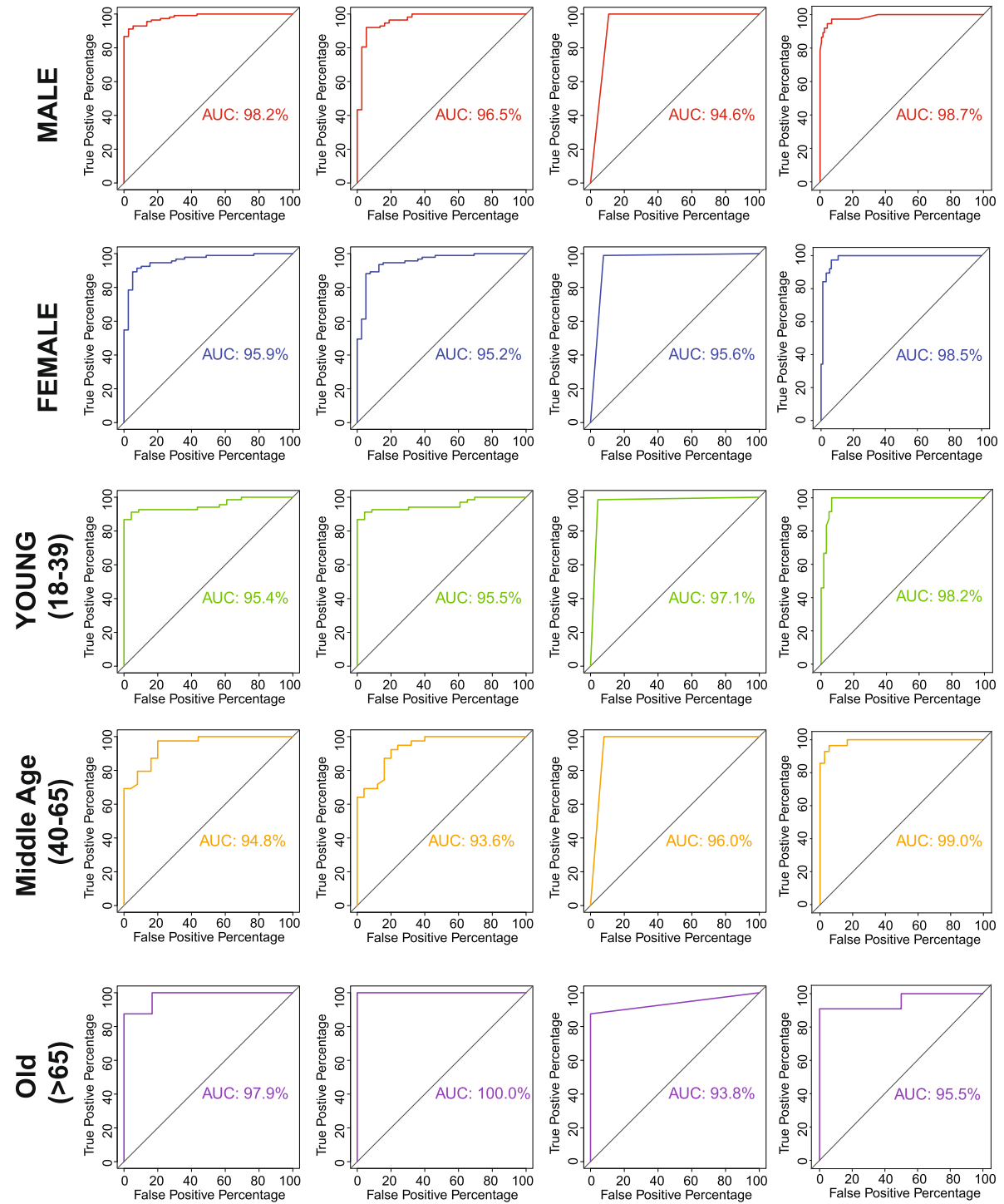

Fig. 6 Classification of COVID-19 vs. influenza patients in different demographic cohorts. RIDGE, LASSO, random forest (RF), and XGBoost classification models were applied to 5 different cohorts of patients

population much more. According to CDC data, people aged 65 or above the only constitute $3.9 \%$ of all influenza cases from 2010 to 2016 [22]. In contrast, people aged 0-4 years made up $13.2 \%$ of influenza cases, and those aged $18-49$ years made up $7.4 \%$ of influenza cases. For COVID-19, however, only around $4 \%$ of cases affect people $0-19$ years of age, and $33 \%$ of cases affect those 60 or above [23].

Despite promising results, several limitations exist for our study, all of which stem from the lack of large-scale clinical data. First, our sample size is severely limited because most clinical reports published do not publish individual-level patient data. Second, data on influenza signs and symptoms are equally inaccessible. We were only able to locate data for patients with H1N1 influenza A, which is not one of the active strains in the current influenza season. Third, many of our data sources are case studies that focused on specific cohorts of COVID19 patients. This increases the chance of us capturing a patient population that is not representative of the general population, although this is an inherent risk of sampling. We anticipate that as more data are made openly available in the weeks and months to come, we will be able to build a more robust computational model. Therefore, we intend to provide the model we constructed as a computational framework for computation-aided diagnosis of COVID-19 data rather than a ready-to-use model. We also encourage researchers around the world to 
release de-identified patient data to aid in data mining and machine learning efforts against COVID-19.

\section{Conclusions}

In conclusion, we demonstrated the use of machine learning models to predict COVID-19 presence using only commonly recorded clinical variables. Specifically, we successfully differentiated COVID-19 patients from influenza A patients using these clinical variables alone. Our machine learning models were constructed using publicly available data in published literature. We also conducted correlational analyses on this dataset and determined that males with COVID-19 have higher serum neutrophil and leukocyte levels than females with COVID-19. Finally, we used this dataset to cluster COVID-19 patients into 3 clinically relevant subtypes.

\section{Supplementary information}

Supplementary information accompanies this paper at https://doi.org/10. 1186/s12911-020-01266-z.

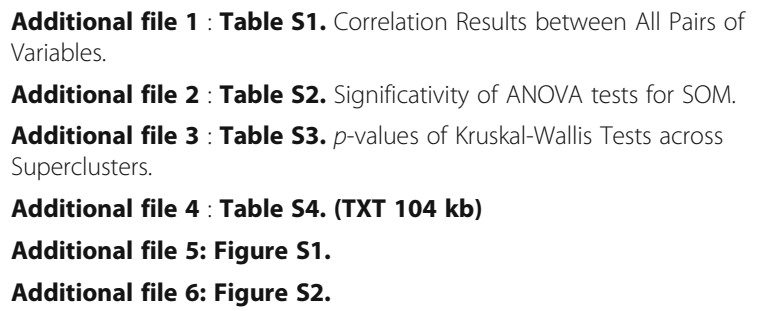

\section{Abbreviations}

CRP: C-reactive Protein; ANOVA: Analysis of Vatriance; SOM: Self-organizing map; XGBoost: Extreme Gradient Boosting; ROC: Receiver Operating Characteristic; AUC: Area Under the Curve; PR: Precision-Recall; PCA: Principle Component Analysis

\section{Acknowledgements}

Not applicable.

\section{Authors' contributions}

Conceptualization, W.M.O.; methodology, W.M.O. and W.T.L.; software, W.T.L. J.M., and N.S.; validation, W.T.L., J.M., and N.S.; formal analysis, W.T.L., J.M., and N.S.; investigation, W.T.L, J.M., N.S., G.C., J.C., J.C.T., L.A., C.O.H., J.X., L.M.W., T.Z., A.L., A.G., and T.K.H.; resources, W.M.O.; data curation, W.T.L, J.M., N.S., G.C., J.C., J.C.T., L.A., C.O.H., J.X., L.M.W., T.Z., A.L., A.G., and T.K.H.; writing-original draft preparation, W.T.L. and N.S.; writing-review and editing, W.M.O., E.Y.C., M.R.R., S.Z.K., and M.A.Y.; visualization, W.T.L., J.M., and N.S.; supervision, W.M.O.; project administration, W.M.O.; funding acquisition, E.Y.C. All authors have read and agreed to the published version of the manuscript.

\section{Funding}

University of California, Office of the President/Tobacco-Related Disease Research Program Emergency COVID-19 Research Seed Funding Grant (ROORG2369) to W.M.O.

\section{Availability of data and materials}

The datasets used for our study can be found in the following repository: https://github.com/yoshihiko1218/COVID19ML. All code for machine learning models used in this study are also included in the above repository.

Ethics approval and consent to participate Not applicable.
Consent for publication

Not applicable.

\section{Competing interests}

The authors declare that they have no competing interests.

\section{Author details}

${ }^{1}$ Department of Surgery, Division of Otolaryngology-Head and Neck Surgery, UC San Diego School of Medicine, San Diego, CA 92093, USA. ${ }^{2}$ Research Service, VA San Diego Healthcare System, San Diego, CA 92161, USA.

${ }^{3}$ Department of Medicine, Columbia University Medical Center, New York, NY 10032, USA. ${ }^{4}$ Department of Internal Medicine, Emory University School of Medicine, Atlanta, GA 30322, USA. ${ }^{5}$ Department of Radiology, University of California San Diego, San Diego, CA 92093, USA. ${ }^{6}$ Radiology Service, VA San Diego Healthcare System, San Diego, CA 92161, USA. Department of Urology, University of California San Diego, San Diego, CA 92093, USA. ${ }^{8}$ Urology Service, VA San Diego Healthcare System, San Diego, CA 92161, USA.

Received: 27 May 2020 Accepted: 16 September 2020

Published online: 29 September 2020

\section{References}

1. Chang MG, Yuan X, Tao Y, Peng X, Wang F, Xie L, Sharma L, Dela Cruz CS, Qin E. Time Kinetics of Viral Clearance and Resolution of Symptoms in Novel Coronavirus Infection. Am J Respir Crit Care Med. 2020;201(9):1150-2.

2. Zhang $M Q$, Wang $X H$, Chen $Y L$, Zhao KL, Cai $Y Q$, An CL, Lin MG, Mu XD. Clinical features of 2019 novel coronavirus pneumonia in the early stage from a fever clinic in Beijing. Zhonghua Jie He He Hu Xi Za Zhi. 2020;43(3):215-8.

3. Feng $K$, Yun YX, Wang XF, Yang GD, Zheng YJ, Lin CM, Wang LF. Analysis of CT features of 15 children with 2019 novel coronavirus infection. Zhonghua Er Ke Za Zhi. 2020;58(0):E007.

4. Li Y, Guo F, Cao Y, Li L, Guo Y. Insight into COVID-2019 for pediatricians. Pediatr Pulmonol. 2020;55:E1-E4.

5. HUANG P. If Most of your coronavirus tests come Back positive, You're not testing enough: NPR; Washington D.C.; 2020.

6. Sun P, Qie S, Liu Z, Ren J, Li K, Xi J. Clinical characteristics of hospitalized patients with SARS-CoV-2 infection: a single arm meta-analysis. J Med Virol. 2020;92(6):612-617.

7. Yang J, Zheng Y, Gou X, Pu K, Chen Z, Guo Q, Ji R, Wang H, Wang Y, Zhou $Y$. Prevalence of comorbidities in the novel Wuhan coronavirus (COVID-19) infection: a systematic review and meta-analysis. Int J Infect Dis. 2020;94:91-5.

8. Cao Y, Liu X, Xiong L, Cai K. Imaging and clinical features of patients with 2019 novel coronavirus SARS-CoV-2: a systematic review and meta-analysis. J Med Virol. 2020;92:1449-59.

9. Cheng Y, Zhao H, Song P, Zhang Z, Chen J, Zhou YH. Dynamic changes of lymphocyte counts in adult patients with severe pandemic H1N1 influenza a. J Infect Public Health. 2019;12(6):878-83.

10. Squires RB, Noronha J, Hunt V, Garcia-Sastre A, Macken C, Baumgarth N, Suarez D, Pickett BE, Zhang Y, Larsen CN, et al. Influenza research database: an integrated bioinformatics resource for influenza research and surveillance. Influenza Other Respir Viruses. 2012;6(6):404-16.

11. Boelaert J, Bendhaiba L, Olteanu M, Villa-Vialaneix N. SOMbrero: an R package for numeric and non-numeric self-organizing map; 2013.

12. Chen T, Carlos G. XGBoost: A Scalable Tree Boosting System. In: KDD '16: Proceedings of the 22nd ACM SIGKDD International Conference on Knowledge Discovery and Data Mining; 2016. p. 9.

13. Kolifarhood G, Aghaali M, Mozafar Saadati H, Taherpour N, Rahimi S, Izadi N, Hashemi Nazari SS. Epidemiological and clinical aspects of COVID-19; a narrative review. Arch Acad Emerg Med. 2020;8(1):e41.

14. Jerez JM, Molina I, Garcia-Laencina PJ, Alba E, Ribelles N, Martin M, Franco L. Missing data imputation using statistical and machine learning methods in a real breast cancer problem. Artif Intell Med. 2010;50(2):105-15.

15. Al'Aref SJ, Maliakal G, Singh G, van Rosendael AR, Ma X, XU Z, Alawamlh OAH, Lee $B$, Pandey M, Achenbach S, et al. Machine learning of clinical variables and coronary artery calcium scoring for the prediction of obstructive coronary artery disease on coronary computed tomography angiography: analysis from the CONFIRM registry. Eur Heart J. 2020;41(3):359-67.

16. Hollingsworth J. A coronavirus test can be developed in 24 hours. So why are some countries still struggling to diagnose? Atlanta: CNN; 2020. 
17. Yong E. How the pandemic will end. Boston: The Atlantic; 2020

18. Molloy EJ, Bearer CF. COVID-19 in children and altered inflammatory responses. Pediatr Res. 2020;88:340-341.

19. Andersen CJ, Vance TM. Gender Dictates the Relationship between Serum Lipids and Leukocyte Counts in the National Health and Nutrition Examination Survey 1999(-)2004. J Clin Med. 2019;8(3):365.

20. Bain BJ, England JM. Normal haematological values: sex difference in neutrophil count. Br Med J. 1975;1(5953):306-9.

21. Wenham C, Smith J, Morgan R, Gender, Group C-W. COVID-19: the gendered impacts of the outbreak. Lancet. 2020;395(10227):846-8

22. Tokars JI, Olsen SJ, Reed C. Seasonal incidence of symptomatic influenza in the United States. Clin Infect Dis. 2018;66(10):1511-8.

23. Malmgren J, Guo B, Kaplan HG. COVID-19 Confirmed Case Incidence Age Shift to Young Persons Age 0-19 and 20-39 Years Over Time: Washington State March-April 2020. MedRxiv. 2020.

\section{Publisher's Note}

Springer Nature remains neutral with regard to jurisdictional claims in published maps and institutional affiliations.

Ready to submit your research? Choose BMC and benefit from:

- fast, convenient online submission

- thorough peer review by experienced researchers in your field

- rapid publication on acceptance

- support for research data, including large and complex data types

- gold Open Access which fosters wider collaboration and increased citations

- maximum visibility for your research: over $100 \mathrm{M}$ website views per year

At BMC, research is always in progress.

Learn more biomedcentral.com/submissions 